\title{
Relação entre os caracteres determinantes das eficiências no uso de nitrogênio e fósforo em milho ${ }^{1}$
}

\author{
Felipe Bermudez Pereira ${ }^{2}$, Júlio César DoVale ${ }^{3}$,Pedro Crescêncio Souza Carneiro ${ }^{4}$, Roberto Fritsche-Neto ${ }^{5}$
}

\section{RESUMO}

O melhoramento genético das eficiências no uso de N (EUN) e P (EUP) é um dos meios para se obterem produtividades de grãos satisfatórias, com menores custos e de modo sustentável. Todavia, pouco se sabe a respeito da relação entre os caracteres determinantes dessas eficiências, o que tem dificultado o uso da seleção precoce e indireta. Portanto, objetivou-se, com este trabalho, identificar a relação entre os caracteres determinantes das eficiências no uso de nitrogênio e fósforo, em milho. Para isso, avaliaram-se 14 linhagens e 39 híbridos simples, em dois experimentos, em baixa e alta disponibilidade de $\mathrm{N}$ e $\mathrm{P}$, em delineamento inteiramente ao acaso, com duas repetições, em esquema fatorial simples. Os experimentos foram conduzidos em telado. Foram utilizados tubos cilíndricos de PVC, com $4 \mathrm{dm}^{3} \mathrm{de}$ capacidade, preenchidos com dois tipos de substrato, de acordo com o experimento. As soluções nutritivas foram fornecidas a partir do sétimo dia após o transplantio, aplicando-se $250 \mathrm{ml}$ tubo ${ }^{-1}$, a cada dois dias. As plantas foram colhidas em estádio de seis folhas completamente expandidas (V6) e os caracteres avaliados foram: massa da parte aérea seca (MPS), área de raiz específica (ARE), comprimento de raízes laterais (CRLat) e axiais (CRAxi) e os dois componentes da EUN e EUP, as eficiências de utilização (EUt) e a de absorção (EAb). Foram realizadas análises de variância e de trilha dos dados coletados. Os caracteres de raiz não apresentaram efeitos significativos sobre as EUN e EUP. A MPS é o principal determinante das EUN e EUP, independentemente da disponibilidade nutricional.

Palavras-chave: Zea mays L., estresses abióticos, seleção indireta, seleção precoce.

\section{ABSTRACT \\ Relationship among determinant characters of nitrogen and phosphorus use efficiency in maize}

The genetic improvement of $\mathrm{N}$ (EUN) and $\mathrm{P}$ (EUP) use efficiency is a less expensive and more sustainable way to obtain satisfactory grain yields. However, the lack of knowledge about the relationship among the determinant characters of nutrient use efficiency has made the use of early and indirect selection difficult. Therefore, the objective of this study was to identify the relationship among the determinant characters of nitrogen and phosphorus use efficiency in maize. In this study, 14 lines and 39 single-cross hybrids were tested in two experiments with low and high $\mathrm{N}$ and $\mathrm{P}$ availability, in a completely randomized simple factorial design with two replications. The experiments were conducted in a shade house. Were used PVC cylindrical tubes with $4 \mathrm{dm}^{3}$ capacity filled with two types of substrate, according to

Recebido para publicação em 07/11/2012 e aprovado em 21/05/2013.

${ }^{1}$ Trabalho extraído de dissertação apresentada ao curso de Pós-Graduação em Genética e Melhoramento pertencente à Universidade Federal de Viçosa. Financiado pela CAPES. ${ }^{2}$ Engenheiro-Agrônomo, Mestre. Departamento de Genética, Escola Superior de Agricultura "Luiz de Queiroz", Avenida Pádua Dias, 11, 13418-900, Piracicaba, São Paulo, Brasil. fbermudez@usp.br (autor para correspondência).

${ }^{3}$ Engenheiro-Agrônomo, Doutor. Departamento de Agronomia, Universidade Federal do Tocantins, Campus Universitário de Gurupi, Rua Badejós, Chácaras 69 e 72, Lote 07, 77402-970, Gurupi, Tocantins, Brasil. juliocvale@gmail.com

${ }^{4}$ Engenheiro-Agrônomo, Doutor. Departamento de Biologia, Universidade Federal de Viçosa, Campus Viçosa, Avenida Peter Henry Rolfs, s/n, 36570-000, Viçosa, Minas Gerais, Brasil. carneiro@ufv.br

${ }^{5}$ Engenheiro-Agrônomo, Doutor. Departamento de Fitotecnia, Universidade Federal de Viçosa, Campus Viçosa, Avenida Peter Henry Rolfs, s/n, 36570-000, Viçosa, Minas Gerais, Brasil. rfritscheneto@gmail.com

Rev. Ceres, Viçosa, v. 60, n.5, p. 636-645, set/out, 2013 
the experiment. The nutrient solutions were provided from the seventh day after transplanting, applying $250 \mathrm{ml}$ tube ${ }^{1}$ every two days. Plants were harvested at the stage of six fully expanded leaves (V6) to evaluate the traits: shoot dry mass, specific root area, lateral and axial root length and the two components of EUN and EUP, i.e., the utilization (EUt) and uptake efficiency (EAb). According to the analyses of variance and path analysis of the collected data, EUN and EUP were not significantly influenced by the root traits. The main determinant character of EUN and EUP is shoot dry mass, regardless of the nutrient availability.

Key words: Zea mays L., abiotic stress, indirect selection, early selection.

\section{INTRODUÇÃO}

A maioria dos ambientes naturais apresenta condições desfavoráveis, em relação aos recursos que limitam a produção agrícola, principalmente no que diz respeito ao N e P (Nielsen et al., 1999). Ademais, o elevado custo com adubação nitrogenada e fosfatada tem inviabilizado, em algumas situações, o aumento da produção dos pequenos agricultores, principalmente em regiões marginais de cultivo. Entretanto, em países desenvolvidos, em que quantidades demasiadas de adubos, principalmente de $\mathrm{N}$, são fornecidas às culturas, ocorrem problemas especialmente nas áreas da saúde e do meio ambiente, em razão do seu grande potencial poluidor (Ahlgren et al., 2008). Adicionalmente, para produzir esses fertilizantes são necessários recursos não renováveis, que se estão tornando cada vez mais escassos (Murrel \& Fixen, 2006; Lopes et al., 2010). Assim, entre os desafios para a produção agrícola, estão o de suprir as crescentes necessidades de alimentos da população mundial e utilizar, de forma mais eficiente, os recursos não renováveis (Borém \& Ramalho, 2011). Nesse contexto, torna-se importante o desenvolvimento de cultivares eficientes no uso de $\mathrm{N}$ e $\mathrm{P}$.

Moll et al. (1982) definiram a eficiência no uso de nutrientes, ou eficiência nutricional, como a relação entre a massa de grãos ou de parte aérea seca por unidade de nutriente disponível. De acordo com esses autores, a eficiência nutricional é constituída por dois componentes: (i) eficiência na absorção, mensurado pela relação entre a quantidade total de nutriente extraído pela planta e a quantidade desse nutriente disponível no solo e, (ii) eficiência na utilização, obtida pela razão entre a massa de grãos ou de parte aérea e a quantidade total de nutriente extraído pela planta. Dessa forma, ganho na eficiência nutricional pode ser alcançado por meio de aumento da eficiência na absorção e, ou, da eficiência na utilização.

O uso de métodos de avaliação precoce ou de seleção indireta em plantas é também de grande interesse, no melhoramento para estresses abióticos, pois acelera o processo de seleção, descartando, de imediato, os genótipos menos promissores, concentrando, assim, os recursos nos potencialmente superiores (Machado et al., 2004). Para isso, o conhecimento da relação entre caracteres é de grande valia, principalmente, se os caracteres de interesse apresentarem baixa herdabilidade e, ou, forem difíceis de mensurar e identificar (Cruz et al., 2004). Contudo, as estimativas de correlações são medidas de associação linear e não permitem tirar conclusões sobre a relação de causa e efeito entre caracteres. Em razão disso, procede-se à análise de trilha, que consiste no estudo dos efeitos diretos e indiretos de caracteres sobre uma variável básica (Wright, 1921).

Em relação ao estádio de avaliação e seleção, sabese que o potencial de produção do milho é definido precocemente, por ocasião da emissão da quarta à sexta folha. O referido estádio é denominado como diferenciação floral, o qual coincide com o término da fase de diferenciação das folhas. Portanto, nessa etapa já são definidos a área foliar (Fischer \& Palmer, 1984) e o número potencial de grãos que a planta deverá apresentar (Coelho et al., 2010).

Dentre os diversos caracteres que podem ser avaliados em condições de estresse abiótico, a massa de parte aérea seca pode ser um indicador de produtividade de grãos (Bignotto et al., 2000). Além disso, sabe-se também que um sistema radicular eficiente é aquele que aperfeiçoa a relação entre a quantidade de recursos adquiridos e empregados para sua obtenção e manutenção (Taiz \& Zeiger, 2009). Nesse sentido, diversos trabalhos buscam relacionar as características do sistema radicular com os componentes da eficiência nutricional (Chun et al., 2005; Liu et al., 2009; Zhu et al., 2006).

O objetivo deste estudo foi identificar a relação entre os caracteres determinantes da eficiência no uso de nitrogênio e fósforo em milho.

\section{MATERIAL E MÉTODOS}

Foram conduzidos dois experimentos, com 39 combinações híbridas experimentais e 14 linhagens de milho, em condições contrastantes de disponibilidades de N (experimento 1) e de $\mathrm{P}$ (experimento 2). Os genótipos utilizados são oriundos do banco de germoplasma do Programa 
Milho ${ }^{\circledR 6}$ e foram identificados como contrastantes para a eficiência no uso de $\mathrm{N}$ e P. Utilizou-se o delineamento inteiramente casualizado, com duas repetições, em esquema fatorial simples (53 genótipos x dois níveis de nutriente). A parcela foi constituída de uma planta por tubo. Os experimentos foram conduzidos em telado, localizado no Campo Experimental Diogo Alves de Mello (2045'14"S; $42^{\circ} 52^{\prime} 53^{\prime \prime} \mathrm{W}, 649$ metros de altitude), pertencente ao Departamento de Fitotecnia da Universidade Federal de Viçosa, Brasil, durante o mês de outubro de 2010.

As sementes foram esterilizadas e, posteriormente, postas a germinar em bandejas de polietileno, em células individuais. Após a emergência, transplantou-se uma plântula de cada genótipo para tubos cilíndricos de PVC, com $4 \mathrm{dm}^{3}$ de capacidade $(10 \mathrm{~cm}$ de diâmetro x $50 \mathrm{~cm}$ de altura), preenchidos com dois tipos de substrato, de acordo com o experimento. $\mathrm{O}$ substrato do experimento 1 foi preparado com a mistura de areia lavada e vermiculita, na proporção de 1:1 (Walk et al., 2006). No experimento 2, o substrato foi preparado com a mistura de $50 \%$ de areia lavada, $37,5 \%$ de vermiculita e $12,5 \%$ de solo (horizonte "B" de Latossolo Vermelho-Amarelo distrófico). O solo foi usado para adsorver o fósforo e não deixá-lo prontamente disponível para as plantas.

No experimento 1, utilizaram-se dois níveis de N, um dos quais simulou as condições ideais de nutrientes, aqui denominado por alta disponibilidade (AN) e, o segundo, por baixa (BN). A solução utilizada foi a descrita por Chun et al. (2005), em que a solução para AN continha 2,0 mmol. $\mathrm{l}^{-1} \mathrm{e}$, a de BN, 0,2 mmol. $\mathrm{l}^{-1}$, ou seja, dez vez menos $\mathrm{N}$ do que em AN. A solução nutritiva foi fornecida a partir do sétimo dia após o transplantio, aplicando-se $250 \mathrm{ml} \mathrm{tubo}^{-1}$, a cada dois dias.

No experimento 2, o $\mathrm{P}$ foi adicionado na forma de superfosfato triplo. Para a alta disponibilidade (AP), misturaram-se $192 \mathrm{mg}$ de P.dm ${ }^{-3}$, ao substrato, e, na baixa (BP), $34 \mathrm{mg} \cdot \mathrm{dm}^{-3}$. Os demais nutrientes foram fornecidos por solução nutritiva de Hoagland \& Arnon (1938), modificada, sem adição de fósforo.

As plantas foram coletadas no estádio vegetativo de seis folhas completamente expandidas (V6) e, na ocasião, a parte aérea foi separada das raízes. Os caracteres mensurados foram: massa da parte aérea seca (MPS), massa de raiz seca (MRS), comprimento de raiz lateral (CRLat), comprimento de raiz axial (CRAxi), área superficial de raiz lateral (ASLat) e área superficial de raiz axial (ASAxi). As massas de parte aérea e de raiz seca foram determinadas após secagem, até peso constante, em estufa, por 72 horas, a $60^{\circ} \mathrm{C}$, com circulação forçada de ar. Os comprimentos e áreas de raiz foram obtidos por meio

${ }^{6}$ Programa de ensino, pesquisa e extensão do Departamento de Fitotecnia da Universidade Federal de Viçosa, Viçosa - MG do analisador de imagens WinRhizo PRO 2009c, acoplado ao scanner profissional Epson Expression XL 10000, equipado com unidade de luz adicional (TPU), conforme descrito por Bouma et al. (2000), utilizando-se, entretanto, a raiz inteira e não apenas amostras. Raízes com diâmetros inferiores ou iguais a $0,5 \mathrm{~mm}$ foram consideradas raízes laterais, e, aquelas com diâmetros acima de 0,5 mm, como raízes axiais (Trachsel et al., 2009). A partir desses valores, foi obtida a área de raiz específica (ARE), pela seguinte expressão:

$A R E=\frac{A S L a t+A S A x}{M R S}$

Para a quantificação do teor de N, retirou-se uma amostra de 0,2 g da massa da parte aérea seca por planta e foram feitas a digestão, a destilação e a titulação, utilizando-se o método descrito por Bremner (1996). Para a quantificação do teor de $\mathrm{P}$, retirou-se uma amostra de 0,1 $\mathrm{g}$ e foram feitas a digestão nitroperclórica e a leitura do teor de P, em espectrofotômetro, a $725 \mathrm{~nm}$ (Malavolta et al., 1989).

Os índices de eficiência nutricional foram obtidos, conforme descrito por Moll et al. (1982), em $m g \cdot \mathrm{mg}^{-1}$ :

$$
\begin{aligned}
& E A b N=\frac{N \text { (planta })}{N(\text { aplicado })} \text { e } E A b P=\frac{P \text { (planta })}{P \text { (aplicado })} \\
& E U t N=\frac{M P S}{N(\text { planta })} \text { e } E U t P=\frac{M P S}{P \text { (planta })} \\
& E U N=\frac{M P S}{N(\text { aplicado })}=E U t N \times E A b N \mathrm{e} \\
& E U P=\frac{M P S}{P(\text { aplicado })}=E U t P \times E A b P
\end{aligned}
$$

Em razão do caráter CRLat não ter apresentado distribuição normal, procedeu-se à transformação de dados, por $\log (x+1)$.

Inicialmente, para todos os caracteres avaliados, foram realizadas as análises de variância, de acordo com o esquema fatorial simples, ou seja, considerando-se os dois níveis de disponibilidade, de $\mathrm{N}$ ou de $\mathrm{P}$, por meio do seguinte modelo matemático:

$$
Y_{i j k}=\mu+g_{i}+a_{j}+\left(g a_{i j}\right)+\varepsilon_{i j k}
$$

em que $Y_{i j k}$ é o valor observado para o caráter no $i$-ésimo genótipo, no j-ésimo nível, na $k$-ésima repetição; $\mu$ é a média geral para o caráter; $g_{i}$ é o efeito do $i$-ésimo genótipo $(i=1,2,3, \ldots, 53)$, considerado como de efeito aleatório, em que g NID $\left(0, \sigma_{\mathrm{g}}^{2}\right)$; $\mathrm{a}_{\mathrm{i}}$ é o efeito da j-ésima dose de nutriente $(j=1,2)$, considerado como de efeito fixo, em que a N $\left(\mathrm{a}, \mathrm{a}^{2}\right) ;\left(\mathrm{ga}_{\mathrm{ij}}\right)$ é o efeito da interação do $i$-ésimo genótipo, com a $j$-ésima dose do nutriente, considerado como efeito aleatório, em que ga NID $\left(0, \sigma_{\mathrm{ga}}^{2}\right) ; \varepsilon_{i j k}$ é o efeito do erro aleatório associado à observação de ordem 
$i j k$, em que $\varepsilon_{i j k} \sim \operatorname{NID}\left(0, \sigma^{2}\right)$. O efeito de genótipos foi considerado como aleatório, pois esses representam uma amostra do germoplasma do Programa Milho ${ }^{\circledR}$ da UFV. Por outro lado, o efeito de níveis de nutriente foi considerado como fixo, pois as inferências e conclusões são específicas para cada condição de cultivo. Com isso, o modelo misto considerado foi analisado por meio de equação de quadrados mínimos, conforme descrito por Barbin (1998).

Para a realização das análises de variância individuais, ou seja, para cada nível de $\mathrm{N}$ ou de $\mathrm{P}$, foi considerado o seguinte o modelo:

$$
\mathrm{Y}_{i k}=\mu+\mathrm{g}_{i}+\varepsilon_{i k}
$$

em que, $\mathrm{Y}_{i k}$ é o valor observado para o caráter, no i-ésimo genótipo, na $k$-ésima repetição; $\mu$ é a média geral para o caráter; $\mathrm{g}_{i}$ é o efeito do $i$-ésimo genótipo, considerado como de efeito aleatório, em que $g \sim \operatorname{NID}\left(0, \sigma_{\mathrm{g}}^{2}\right) ; \varepsilon_{i k}$ é o efeito do erro (aleatório) associado à observação de ordem $i k$, em que $\varepsilon \sim \operatorname{NID}\left(0, \sigma_{\mathrm{g}}^{2}\right)$.

As estimativas das correlações fenotípicas foram obtidas para todos os caracteres. Posteriormente, a partir desses valores, foi realizada a diagnose de multicolinearidade entre os caracteres explicativos e seu grau na matriz de correlação fenotípica foi estabelecido com base no número de condição (NC) (Montgomery \& Peck, 1981). O desdobramento das correlações fenotípicas, em efeitos diretos e indiretos dos caracteres explicativos sobre a EU (caráter dependente), foi feito por meio da análise de trilha (Wright, 1921).

As análises de variância foram realizadas com a utilização do programa estatístico computacional Statistical Analysis System (SAS) versão 9.1 (SAS Institute, 2003). As diagnoses de multicolinearidade e as análises de trilha foram realizadas com auxílio do
Programa Genes - Aplicativo Computacional em Genética e Estatística (Cruz, 2006).

\section{RESULTADOS E DISCUSSÃO}

Os dados foram analisados por meio do procedimento UNIVARIATE (SAS, 2003), para determinar se os erros experimentais das variáveis apresentavam distribuição normal de probabilidade e homogeneidade de variância (dados não mostrados).

$\mathrm{Na}$ análise de variância para o experimento de N, verificou-se ocorrência de diferenças significativas, entre genótipos, para todos os caracteres estudados; os níveis considerados desse nutriente foram suficientemente contrastantes para todos os caracteres, exceto para CRLat (Tabela 1). No entanto, apenas para os caracteres MPS e EUN os genótipos apresentaram comportamento diferencial, em função da disponibilidade $\operatorname{de} \mathrm{N}(\mathrm{G} \times \mathrm{N})$.

No experimento de $\mathrm{P}$, somente não houve diferença significativa entre genótipos para o caráter EUtP. De modo semelhante ao ocorrido no experimento anterior, foi observado que os níveis considerados de $\mathrm{P}$ foram suficientemente contrastantes para discriminar os genótipos para a maioria dos caracteres, sendo as exceções ARE, CRAxi e EAbP (Tabela 2). Esse resultado para EAbP pode ser, em parte, explicado pela diminuição proporcional do $\mathrm{P}$ aplicado e da concentração do P na planta, em BP, o que pode ter sido ocasionado pela adsorção de maior percentagem do P aplicado nesse nível. Ademais, para os caracteres MPS, CRLat, CRAxi e EUP, os genótipos apresentaram comportamento diferencial, em função da disponibilidade de $\mathrm{P}(\mathrm{G} \times \mathrm{P})$.

Os coeficientes de variação experimental apresentaram valores dentro do aceitável para esse tipo de estudo (Bänziger \& Lafitte, 1997; Machado et al., 2004; Fritsche-

Tabela 1. Resumo das análises da variância para os caracteres massa de parte aérea seca (MPS), área de raiz específica (ARE), comprimento de raiz lateral (CRLat) e axial (CRAxi), eficiência na absorção (EAbN), utilização (EUtN) e no uso de nitrogênio (EUN) em 14 linhagens e 39 combinações híbridas de milho, avaliados em dois níveis de N, Viçosa-MG, 2010

\begin{tabular}{|c|c|c|c|c|c|c|c|c|}
\hline \multirow{2}{*}{ FV } & \multirow{2}{*}{ GL } & \multicolumn{7}{|c|}{ Quadrado Médio } \\
\hline & & MPS & ARE & CRLat & CRaxi & EAbN & EUtN & EUN \\
\hline Genótipos (G) & 52 & $0,17^{* *}$ & $4 \mathrm{E}-4^{* *}$ & $0,12^{* * *}$ & $25,68^{* *}$ & $0,01^{* *}$ & $24,32^{\text {ns }}$ & $15,06^{* *}$ \\
\hline Níveis de N (N) & 1 & $8,78^{* *}$ & $3 \mathrm{E}-3^{* *}$ & $0,05^{\mathrm{ns}}$ & $34,65^{* *}$ & $2,33^{* *}$ & $4968,41^{* *}$ & $3362,24^{* *}$ \\
\hline $\mathrm{GxN}$ & 52 & $0,04^{* *}$ & $2 \mathrm{E}-4^{\mathrm{ns}}$ & $0,01^{\mathrm{ns}}$ & $2,95^{\mathrm{ns}}$ & $4 \mathrm{E}-3^{\mathrm{ns}}$ & $21,78^{\text {ns }}$ & $6,54^{* *}$ \\
\hline Resíduo & 104 & 0,02 & $2 \mathrm{E}-4$ & 0,01 & 3,09 & $3 \mathrm{E}-3$ & 20,73 & 3,24 \\
\hline Média & & 0,60 & 0,12 & 1,24 & 7,50 & 0,19 & 29,30 & 6,07 \\
\hline $\mathrm{CV}(\%)$ & & 24,87 & 11,56 & 9,49 & 23,44 & 30,60 & 15,54 & 29,63 \\
\hline$\widehat{\sigma}_{g}^{2}$ & & 0,04 & 5E-5 & 0,03 & 5,65 & $3 \mathrm{E}-3$ & 0,90 & 2,96 \\
\hline$\hat{\sigma}_{g a}^{2}$ & & $5 \mathrm{E}-3$ & $4 \mathrm{E}-6$ & 0 & 0 & $3 \mathrm{E}-4$ & 0,26 & 0,82 \\
\hline$\hat{h}_{g}^{2}$ & & 0,87 & 0,52 & 0,88 & 0,88 & 0,75 & 0,15 & 0,79 \\
\hline
\end{tabular}

${ }^{\text {ns }}$ não significativo $\mathrm{e}^{* *}$ significativo a 0,01 de probabilidade pelo teste F. $l=a / a-1$ 
Neto et al., 2010), o que indica boa precisão experimental e confiabilidade das estimativas.

Nas análises individuais de $\mathrm{N}$, detectaram-se diferenças significativas entre os genótipos, para a maioria dos caracteres estudados, em alta e baixa disponibilidade (Tabela 3). A exceção foi a EUtN, nos dois níveis, e ARE, em BN. As diferenças genéticas significativas observadas indicam a existência de variabilidade genética para eficiência nutricional, o que possibilita a seleção e os ganhos genéticos dentro de cada nível de N (DoVale et al., 2012; Oliveira et al., 2013).

As médias, as variâncias genéticas e a herdabilidade dos caracteres foram menores em BN. Isso porque, em condições de estresse, há limitação do crescimento e redução da expressão da variabilidade genética, pois os genótipos tendem a ter desempenhos semelhantes, dificultando, assim, a seleção (Coque \& Gallais, 2006). As exceções foram EAbN e EUN. Elas apresentaram maior variabilidade genética, quando em baixa disponibilidade. Isso indica que o estresse por $\mathrm{N}$ possibilita que os genótipos expressem a variabilidade desses caracteres, proporcionando a seleção dos mais eficientes. Entretanto, a herdabilidade dos caracteres foi menor, em BN, o que indica que os demais componentes também aumentaram e em maior magnitude do que a variância genética. A média é maior em baixa disponibilidade pelo fato de a biomassa não diminuir na mesma proporção da disponibilidade dos nutrientes, ou seja, o milho diminui a concentração do nutriente na planta, com o mínimo de redução na biomassa (Hirel et al., 2007).

A redução da $\mathrm{ARE}$, em $\mathrm{BN}$, em relação à redução, em AN, contradiz o observado por Chun et al. (2005), quando afirma que genótipos de milho respondem à deficiência de $\mathrm{N}$ pelo aumento de alocação de carbono da parte aérea para a formação de raízes, visando ao aumento da sua área superficial. Porém, não há necessidade do inves- timento em ARE para o $\mathrm{N}$, já que o $\mathrm{N}$ é absorvido, juntamente com a água, por fluxo de massa, não necessitando da interceptação radicular.

No experimento de $\mathrm{P}$, foram verificadas diferenças significativas entre os genótipos, para a maioria dos caracteres estudados, em alta e em baixa disponibilidades (Tabela 4). A exceção foi a EUtP, nos dois níveis. Assim como observado por Brito (2009), o CRLat dos genótipos aumentou, em BP, em relação ao AP. Isso indica que as plantas investem em comprimento de raízes de menor diâmetro, quando em estresse, por deficiência desse nutriente. O P, diferentemente do $\mathrm{N}$, é absorvido por difusão, o que exige grande proximidade entre a raiz e o nutriente no solo, fazendo com que maior CRLat constitua ponto chave na habilidade das plantas para adquirir $\mathrm{P}$ do solo (Parentoni et al., 2011).

As variâncias genéticas dos caracteres de raiz e da EUP apresentaram maior valor, quando em baixa disponibilidade de P. Isso indica que o estresse por P, assim como o por $\mathrm{N}$, permite que todos os caracteres de raiz expressem sua variabilidade. Já para MPS e EAbP, efeito contrário foi observado.

Para alcançar aumentos na EU de $\mathrm{N}$ ou de $\mathrm{P}$, é necessário o aumento de seus componentes (Parentoni et al., 2011; Hirel et al., 2007). Segundo Corrales et al. (2007), quando a disponibilidade de um determinado nutriente é limitante, a EAb é mais importante que a EUt. A não significância da EUt em milho pode ser explicada pela menor redução na MPS, em relação ao teor de nutrientes nos tecidos. Com isso, há pouca redução na biomassa, enquanto a concentração do nutriente na planta reduz-se consideravelmente, fazendo que não haja grande diferença entre os genótipos.

Nas matrizes de correlação fenotípica, foi diagnosticada a presença de multicolinearidade, com nível modera-

Tabela 2. Resumo das análises da variância para os caracteres massa de parte aérea seca (MPS), área de raiz específica (ARE), comprimento de raiz lateral (CRLat) e axial (CRAxi), eficiência na absorção (EAbP), utilização (EUtP) e no uso de fósforo (EUP) em 14 linhagens e 39 combinações híbridas de milho, avaliados em dois níveis de P, Viçosa-MG, 2010

\begin{tabular}{lrccccccc}
\hline \multirow{2}{*}{ FV } & GL & \multicolumn{7}{c}{ Quadrado Médio } \\
\cline { 3 - 9 } & & MPS & ARE & CRLat & CRaxi & EAbP & EUtP & EUP \\
\hline Genótipos (G) & 52 & $1,27^{* *}$ & $3 \mathrm{E}-4^{* *}$ & $0,06^{* *}$ & $33,72^{* *}$ & $1 \mathrm{E}-4^{* *}$ & $6298,64^{\text {ns }}$ & $12,89^{* *}$ \\
Níveis de P(P) & 1 & $66,94^{* *}$ & $4 \mathrm{E}-4^{\text {ns }}$ & $0,14^{* *}$ & $6,33^{\text {ns }}$ & $7 \mathrm{E}-5^{\text {ns }}$ & $5431465,31^{* *}$ & $990,45^{* *}$ \\
GxP & 52 & $0,38^{* *}$ & $1 \mathrm{E}-4^{\text {ns }}$ & $0,01^{* *}$ & $5,12^{*}$ & $4 \mathrm{E}-5^{\text {ns }}$ & $7885,72^{\text {ns }}$ & $4,24^{* *}$ \\
Resíduo & 104 & 0,16 & $1 \mathrm{E}-4$ & $7 \mathrm{E}-3$ & 3,21 & $4 \mathrm{E}-5$ & 5410,87 & 2,41 \\
\hline Média & & 1,56 & 0,10 & 1,45 & 10,58 & 0,02 & 343,75 & 5,00 \\
CV(\%) & 25,72 & 9,63 & 5,69 & 16,95 & 38,68 & 21,40 & 31,07 \\
$\hat{\sigma}_{g}^{2}$ & & 0,28 & $5 \mathrm{E}-5$ & 0,01 & 7,63 & $2 \mathrm{E}-5$ & 221,94 & 2,62 \\
$\hat{\sigma}_{g a}^{2}$ & & 0,05 & $6 \mathrm{E}-6$ & $1 \mathrm{E}-3$ & 0,48 & $3 \mathrm{E}-7$ & 618,71 & 0,46 \\
$\hat{h}_{g}^{2}$ & & 0,87 & 0,68 & 0,89 & 0,90 & 0,70 & 0,14 & 0,81 \\
\hline
\end{tabular}

ns não significativo, ${ }^{*}$ significativo a $5 \%$ e ${ }^{* *}$ significativo a 0,01 de probabilidade pelo teste F. $l=a / a-1$ 
Tabela 3. Resumo das análises de variância para os caracteres massa de parte aérea seca (MPS), área de raiz específica (ARE), comprimento de raiz lateral (CRLat) e axial (CRAxi), eficiência na absorção (EAbN), utilização (EUtN) e no uso de nitrogênio (EUN) em 14 linhagens e 39 combinações híbridas de milho, avaliados em alta e baixa disponibilidade de N, Viçosa-MG, 2010

\begin{tabular}{|c|c|c|c|c|c|c|c|c|c|c|c|c|c|c|c|}
\hline \multirow{3}{*}{ FV } & \multirow{3}{*}{ GL } & & & \multicolumn{12}{|c|}{ Quadrado Médio } \\
\hline & & \multicolumn{2}{|c|}{ MPS } & \multicolumn{2}{|c|}{ ARE } & \multicolumn{2}{|c|}{ CRLat } & \multicolumn{2}{|c|}{ CRaxi } & \multicolumn{2}{|c|}{ EAbN } & \multicolumn{2}{|c|}{ EUtN } & \multicolumn{2}{|c|}{ EUN } \\
\hline & & Alto & Baixo & Alto & Baixo & Alto & Baixo & Alto & Baixo & Alto & Baixo & Alto & Baixo & Alto & Baixo \\
\hline Genótipos & 52 & $0,18^{* *}$ & $0,03^{* *}$ & $4 \mathrm{E}-4^{*}$ & $2 \mathrm{E}-4^{\mathrm{ns}}$ & $0,08^{* *}$ & $0,05^{* *}$ & $16,35^{* *}$ & $12,27^{* *}$ & $2 \mathrm{E}-3^{* *}$ & $0,02^{* *}$ & $7,03^{\mathrm{ns}}$ & $39,07^{\mathrm{ns}}$ & $1,19^{* *}$ & $20,41^{* *}$ \\
\hline Resíduo & 52 & 0,04 & 0,01 & $2 \mathrm{E}-4$ & $2 \mathrm{E}-4$ & 0,02 & 0,01 & 2,99 & 3,20 & $5 \mathrm{E}-4$ & $6 \mathrm{E}-3$ & 6,49 & 34,98 & 0,23 & 6,25 \\
\hline Média & & 0,81 & 0,40 & 0,12 & 0,11 & 1,26 & 1,23 & 7,91 & 7,10 & 0,08 & 0,30 & 24,42 & 34,19 & 2,05 & 10,09 \\
\hline $\mathrm{CV}(\%)$ & & 23,23 & 24,77 & 12,11 & 10,87 & 9,85 & 9,10 & 21,85 & 25,20 & 26,20 & 26,80 & 10,43 & 17,30 & 23,24 & 24,77 \\
\hline$\hat{\sigma}_{g}^{2}$ & & 0,07 & 0,01 & $9 \mathrm{E}-5$ & $2 \mathrm{E}-5$ & 0,03 & 0,02 & 6,68 & 4,54 & $1 \mathrm{E}-3$ & $5 \mathrm{E}-3$ & 0,27 & 2,05 & 0,48 & 7,08 \\
\hline$\hat{h}_{g}^{2}$ & & 0,81 & 0,69 & 0,45 & 0,21 & 0,81 & 0,75 & 0,82 & 0,74 & 0,77 & 0,59 & 0,08 & 0,10 & 0,81 & 0,69 \\
\hline
\end{tabular}

ns não significativo, * significativo a $5 \%$ e ** significativo a 0,01 de probabilidade pelo teste $\mathrm{F}$

Tabela 4. Resumo das análises de variância para os caracteres massa de parte aérea seca (MPS), área de raiz específica (ARE), comprimento de raiz lateral (CRLat) e axial (CRAxi), eficiência na absorção (EAbP), utilização (EUtP) e no uso de fósforo (EUP) em 14 linhagens e 39 combinações híbridas de milho, avaliados em alta e baixa disponibilidade de P, Viçosa-MG, 2010

\begin{tabular}{|c|c|c|c|c|c|c|c|c|c|c|c|c|c|c|c|}
\hline \multirow{3}{*}{ FV } & \multirow{3}{*}{ GL } & \multicolumn{14}{|c|}{ Quadrado Médio } \\
\hline & & \multicolumn{2}{|c|}{ MPS } & \multicolumn{2}{|c|}{ ARE } & \multicolumn{2}{|c|}{ CRLat } & \multicolumn{2}{|c|}{ CRaxi } & \multicolumn{2}{|c|}{ EAbP } & \multicolumn{2}{|c|}{ EUtP } & \multicolumn{2}{|c|}{ EUP } \\
\hline & & Alto & Baixo & Alto & Baixo & Alto & Baixo & Alto & Baixo & Alto & Baixo & Alto & Baixo & Alto & Baixo \\
\hline Genótipos & 52 & $1,42^{* *}$ & $0,26^{* *}$ & $2 \mathrm{E}-4^{*}$ & $2 \mathrm{E}-4^{* *}$ & $0,03^{* *}$ & $0,04^{* *}$ & $18,26^{* *}$ & $20,07^{* *}$ & $8 \mathrm{E}-5^{* *}$ & $8 \mathrm{E}-5^{*}$ & $2093,41^{\mathrm{ns}}$ & $11643,23^{\text {ns }}$ & $2,41^{* *}$ & $14,36^{* *}$ \\
\hline Resíduo & 52 & 0,24 & 0,08 & $1 \mathrm{E}-4$ & $9 \mathrm{E}-5$ & $9 \mathrm{E}-3$ & $4 \mathrm{E}-3$ & 2,56 & 3,89 & $3 \mathrm{E}-5$ & $5 \mathrm{E}-5$ & 2362,97 & 8580,68 & 0,40 & 4,51 \\
\hline Média & & 2,12 & 0,98 & 0,11 & 0,10 & 1,42 & 1,48 & 10,35 & 10,80 & 0,02 & 0,01 & 180,40 & 510,26 & 2,76 & 7,28 \\
\hline $\mathrm{CV}(\%)$ & & 22,88 & 29,16 & 10,17 & 9,00 & 6,79 & 4,39 & 15,47 & 18,25 & 31,86 & 45,45 & 26,95 & 18,15 & 22,88 & 29,16 \\
\hline$\hat{\sigma}_{g}^{2}$ & & 0,59 & 0,09 & $5 \mathrm{E}-5$ & $7 \mathrm{E}-5$ & 0,01 & 0,02 & 7,85 & 8,09 & $3 \mathrm{E}-5$ & $2 \mathrm{E}-5$ & 0 & 1531,27 & 1,00 & 4,93 \\
\hline$\hat{h}_{g}^{2}$ & & 0,83 & 0,69 & 0,48 & 0,61 & 0,72 & 0,89 & 0,86 & 0,81 & 0,68 & 0,40 & 0 & 0,26 & 0,83 & 0,69 \\
\hline
\end{tabular}

\footnotetext{
ns não significativo, ${ }^{*}$ significativo a $5 \%$ e ${ }^{* *}$ significativo a 0,01 de probabilidade pelo teste $\mathrm{F}$
} 
do (NC: 451,36), entre os caracteres explicativos referentes ao AN. Como todos os caracteres são importantes e não poderiam ser eliminados, a análise de trilha para esse nível de $\mathrm{N}$ foi realizada sob multicolinearidade. Nessa análise, não ocorreram valores de inflação das variâncias (FIVs) superiores a dez (Tabela 5). Isso indica que os coeficientes foram pouco influenciados pela multicolinearidade. Além disso, o valor da constante $k$, escolhido para a estabilização das estimativas, foi pequeno $(0,077)$, ocasionando pouco viés na análise de regressão (Cruz et al., 2004). Os coeficientes de determinação de todas as análises de trilha apresentaram valores elevados (acima de 0,9$)$, o que indica que grande parte da variação do caráter principal foi determinada pelos caracteres explicativos.

Os principais determinantes, em AN, da EUN, foram MPS e EAbN (Tabela 5). Eles apresentaram elevada correlação, porém seus efeitos diretos foram de baixa magnitude. Além disso, a EAbN apresentou efeito indireto, via MPS maior do que seu efeito direto sobre EUN. A ARE apresentou baixa correlação e baixo efeito direto sobre a EUN, indicando que o investimento de carbono em raízes de menor diâmetro não influencia na EUN. Os caracteres CRAxi e CRLat apresentaram correlações de alta magnitu-

Tabela 5. Estimativas dos efeitos diretos e indiretos, que envolvem a variável principal eficiência do uso do nitrogênio (EUN) e as variáveis independentes explicativas: comprimento de raiz lateral (CRLat) e axial (CRAxi), área de raiz específica (ARE), eficiência de absorção de $\mathrm{N}$ (EAbN) e massa da parte área seca (MPS) de 14 linhagens e 39 combinações híbridas de milho avaliados em alto e baixo N, Viçosa, MG, 2010

\begin{tabular}{|c|c|c|c|c|}
\hline \multirow{2}{*}{ Caráter } & \multirow{2}{*}{ Efeitos de Associação } & \multicolumn{3}{|c|}{ Estimativas } \\
\hline & & Alto N & FIV* & Baixo N \\
\hline \multirow{6}{*}{ MPS } & Direto sobre EUN & 0,430 & 7,573 & 0,872 \\
\hline & Indireto via ARE & $-0,001$ & 0,003 & - \\
\hline & Indireto via CRLat & 0,079 & 2,241 & 0,037 \\
\hline & Indireto via CRAxi & 0,099 & 2,241 & 0,014 \\
\hline & Indireto via $\mathrm{EAbN}$ & 0,349 & 4,658 & 0,068 \\
\hline & Total & 0,990 & & 0,990 \\
\hline \multirow{6}{*}{ ARE } & Direto sobre EUN & $-0,025$ & 1,122 & - \\
\hline & Indireto via MPS & 0,022 & 0,017 & - \\
\hline & Indireto via CRLat & 0,006 & 0,015 & - \\
\hline & Indireto via CRAxi & 0,001 & 0,001 & - \\
\hline & Indireto via $\mathrm{EAbN}$ & 0,048 & 0,087 & - \\
\hline & Total & 0,051 & & - \\
\hline \multirow{6}{*}{ CRLat } & Direto sobre EUN & 0,093 & 3,588 & 0,041 \\
\hline & Indireto via MPS & 0,366 & 4,729 & 0,776 \\
\hline & Indireto via ARE & $-0,002$ & 0,005 & - \\
\hline & Indireto via CRAxi & 0,099 & 2,226 & 0,014 \\
\hline & Indireto via $\mathrm{EAbN}$ & 0,288 & 3,162 & 0,059 \\
\hline & Total & 0,851 & & 0,890 \\
\hline \multirow{6}{*}{ CRAxi } & Direto sobre EUN & 0,117 & 3,578 & 0,016 \\
\hline & Indireto via MPS & 0,367 & 4,745 & 0,743 \\
\hline & Indireto via ARE & $-0,003$ & 0,002 & - \\
\hline & Indireto via CRLat & 0,079 & 2,232 & 0,036 \\
\hline & Indireto via $\mathrm{EAbN}$ & 0,282 & 3,029 & 0,057 \\
\hline & Total & 0,853 & & 0,852 \\
\hline \multirow{6}{*}{ EAbN } & Direto sobre EUN & 0,361 & 5,785 & 0,073 \\
\hline & Indireto via MPS & 0,416 & 6,098 & 0,809 \\
\hline & Indireto via ARE & $-0,003$ & 0,017 & - \\
\hline & Indireto via CRLat & 0,074 & 1,961 & 0,033 \\
\hline & Indireto via CRAxi & 0,091 & 1,873 & 0,013 \\
\hline & Total & 0,967 & & 0,928 \\
\hline \multicolumn{2}{|c|}{ Coeficiente de determinação } & 0,952 & & 0,981 \\
\hline \multicolumn{2}{|c|}{ Valor de k usado na análise } & 0,077 & & - \\
\hline \multicolumn{2}{|c|}{ Efeito da variável residual } & 0,218 & & 0,137 \\
\hline \multicolumn{2}{|c|}{ Determinante da matriz } & 0,030 & & - \\
\hline
\end{tabular}

*Fatores que inflacionam as variâncias.

Rev. Ceres, Viçosa, v. 60, n.5, p. 636-645, set/out, 2013 
de com EUN, porém seus efeitos diretos foram inferiores ao valor da variável residual. Contudo, seus efeitos indiretos, via MPS e EAbN, foram altos, corroborando a maior importância desses últimos caracteres.

Em BN, apenas MPS apresentou elevada correlação e elevado efeito direto sobre a EUN. A EAbN apresentou elevada correlação com EUN, mas teve efeito direto inferior ao valor da variável residual. Os caracteres de raiz, de modo semelhante aos observados em AN, apresentaram baixo efeito sobre EUN. Baixos efeitos diretos dos comprimentos radiculares também foram observados por DeLima (2010), indicando que a avaliação desses efeitos diretos dos comprimentos não é importante na seleção de genótipos com maior EUN, em plântulas de milho. Entre- tanto, esses resultados contradizem Liu et al. (2009), que afirmam que, em BN, as plantas investem em maior sistema radicular para suplantar o estresse.

No experimento de $\mathrm{P}$, o principal determinante da EUP foi a MPS, em ambos os níveis (Tabela 6). Os caracteres EAbP, CRLat e CRAxi apresentaram elevada correlação com EUP, porém seus efeitos diretos foram baixos. Entretanto, seus efeitos indiretos, via MPS, foram altos, corroborando a maior importância desse último caráter na determinação da EUP, nesse ambiente, e indicando que a seleção em MPS aumentará esses caracteres. Porém, com o efeito indireto de ARE, via MPS negativo, sua seleção acarretaria diminuição daquele. Esse resultado contradiz Taiz \& Zieger (2009),

Tabela 6. Estimativas dos efeitos diretos e indiretos, que envolvem a variável principal eficiência no uso do fósforo (EUP) e as variáveis independentes explicativas: comprimento de raiz lateral (CRLat) e axial (CRAxi), área de raiz específica (ARE), eficiência na absorção de $\mathrm{P}$ (EAbP) e massa da parte área seca (MPS) de 14 linhagens e 39 combinações híbridas de milho avaliados em alto e baixo P, Viçosa, MG, 2010

\begin{tabular}{|c|c|c|c|}
\hline \multirow{2}{*}{ Caráter } & \multirow{2}{*}{ Efeitos de Associação } & \multicolumn{2}{|c|}{ Estimativas } \\
\hline & & Alto $P$ & Baixo $\mathbf{P}$ \\
\hline \multirow{6}{*}{ MPS } & Direto sobre EUP & 0,926 & 0,884 \\
\hline & Indireto via ARE & 0,005 & 0,003 \\
\hline & Indireto via CRLat & $-1 \mathrm{E}-4$ & 0,007 \\
\hline & Indireto via CRAxi & 0,021 & 0,031 \\
\hline & Indireto via EAbP & 0,039 & 0,065 \\
\hline & Total & 0,990 & 0,990 \\
\hline \multirow{6}{*}{ ARE } & Direto sobre EUP & $-0,010$ & $-0,007$ \\
\hline & Indireto via MPS & $-0,445$ & $-0,318$ \\
\hline & Indireto via CRLat & $7 \mathrm{E}-5$ & $-0,003$ \\
\hline & Indireto via CRAxi & $-0,013$ & $-0,016$ \\
\hline & Indireto via EAbP & $-0,013$ & $-0,015$ \\
\hline & Total & $-0,481$ & $-0,360$ \\
\hline \multirow{6}{*}{ CRLat } & Direto sobre EUP & $-2 \mathrm{E}-4$ & 0,009 \\
\hline & Indireto via MPS & 0,666 & 0,641 \\
\hline & Indireto via ARE & 0,004 & 0,002 \\
\hline & Indireto via CRAxi & 0,022 & 0,030 \\
\hline & Indireto via EAbP & 0,028 & 0,043 \\
\hline & Total & 0,719 & 0,725 \\
\hline \multirow{6}{*}{ CRAxi } & Direto sobre EUP & 0,026 & 0,037 \\
\hline & Indireto via MPS & 0,765 & 0,742 \\
\hline & Indireto via ARE & 0,005 & 0,003 \\
\hline & Indireto via CRLat & $-2 \mathrm{E}-4$ & 0,007 \\
\hline & Indireto via EAbP & 0,031 & 0,049 \\
\hline & Total & 0,826 & 0,839 \\
\hline \multirow{6}{*}{ EAbP } & Direto sobre EUP & 0,044 & 0,072 \\
\hline & Indireto via MPS & 0,809 & 0,799 \\
\hline & Indireto via ARE & 0,003 & 0,002 \\
\hline & Indireto via CRLat & $-1 E-4$ & 0,006 \\
\hline & Indireto via CRAxi & 0,018 & 0,025 \\
\hline & Total & 0,874 & 0,903 \\
\hline \multicolumn{2}{|c|}{ Coeficiente de determinação } & 0,981 & 0,981 \\
\hline \multicolumn{2}{|c|}{ Efeito da variável residual } & 0,139 & 0,137 \\
\hline
\end{tabular}


que afirmam que a absorção do P está ligada à área de raiz. Os caracteres de raiz não apresentaram efeitos sobre a EUP, mesmo com o aumento da média do CRLat, em BP. Isso indica que a avaliação desses caracteres também não é importante na seleção de genótipos com maior EUP, em plântulas de milho.

A relação entre MPS e EU evidencia que não basta a planta absorver o nutriente em grande quantidade, mas deve, também usá-lo com eficiência, transformando-o em área fotossintetizante e em órgãos reprodutivos.

\section{CONCLUSÕES}

A massa de parte aérea seca é o caráter determinante da eficiência no uso de nitrogênio e fósforo, independentemente da disponibilidade desses nutrientes.

Em alta disponibilidade de nitrogênio, a eficiência de absorção também contribuiu, embora em menor proporção, para a explicação da eficiência no uso de nitrogênio.

\section{REFERÊNCIAS}

Ahlgren S, Barky A, Bernesson S, Nordberg A, Norén O \& Hansson PA (2008) Ammonium nitrate fertilizer production based on biomass - environmental effects from a life cycle perspective. Bioresource Technology, 99:8034-8041.

Bänziger M \& Lafitte HR (1997) Efficiency of secondary traits for improving maize for low nitrogen target environments. Crop Science, 37:1110-1117.

Barbin D (1998) Componentes de variância: teoria e aplicações. $2^{\mathrm{a}}$ ed. Piracicaba, FEALQ. 120p.

Bignotto EA, Souza EA de, Ramalho MAP, Souza JC de \& Ribeiro PHE (2000) Desempenho de cultivares modernos de milho em relação a cultivares primitivos em uso no sul de Minas Gerais. In: XXIII Congresso Brasileiro de Milho e Sorgo, Uberlândia. Anais, SBMS. CD-ROM.

Borém A \& Ramalho MAP (2011) Estresses abióticos: desafios do melhoramento de plantas nas próximas décadas. In: FritscheNeto R \& Borém A (Eds.) Melhoramento de plantas para condições de estresses abióticos. Visconde do Rio Branco, Editora Suprema. p.09-28.

Bouma TJ, Nielson KL \& Koutstaal BAS (2000) Sample preparation and scanning protocol for computerized analysis of root length and diameter. Plant and Soil, 218:185-196.

Bremner JM (1996) Nitrogen total. In: Page AL (Ed.) Methods of soil analysis. Madison, Soil Science Society of America Press. p. 1085-1121.

Brito CM (2009) Variabilidade genética e caracterização do sistema radical de plântulas de milho para eficiência na absorção e utilização de fósforo. Dissertação de Mestrado. Universidade Federal de Viçosa, Viçosa. 39p.

Chun L, Mi G, Li J, Chen F \& Zhang F (2005) Genetic analysis of maize root characteristics in response to low nitrogen stress. Plant and Soil, 276:369-382.

Coelho AM, França GE de, Pitta GVE, Alves VMC \& Hernani LC (2010) Cultivo do Milho: Nutrição e adubação do milho. Disponível em: http://sistemasdeproducao.cnptia.embrapa.br/ FontesHTML/Milho/CultivodoMilho_2ed/feraduba.htm. Acessado em: 25 de setembro de 2011.
Coque M \& Gallais A (2006) Genomic regions involved in response to grain yield selection at high and low nitrogen fertilization in maize. Theoretical and Applied Genetics, 112:1205-1220.

Corrales I, Amenós M, Poschenrieder C \& Barceló J (2007) Phosphorus efficiency and root exudates in two contrasting tropical maize varieties. Journal of Plant Nutrition, 30:887-900.

Cruz CD (2006) Programa Genes: Biometria. Viçosa, Editora UFV. $382 \mathrm{p}$.

Cruz CD, Regazzi AJ \& Carneiro PCS (2004) Modelos biométricos aplicados ao melhoramento genético. Viçosa, Editora UFV. $480 \mathrm{p}$.

DeLima RO (2010) Herança e relação entre caracteres associados à morfologia de raiz e eficiência de uso de nitrogênio em milho. Dissertação de Mestrado. Universidade Federal de Viçosa, Viçosa. 35 p.

DoVale JC, Fritsche Neto R, Bermudez F \& Miranda GV (2012) Efeitos gênicos de caracteres associados à eficiência no uso de nitrogênio em milho. Pesquisa Agropecuária Brasileira, 47:385392.

Fischer KS \& Palmer FE (1984) Tropical maize. In: Goldsworthy PR \& Fisher NM (Eds.) The physiology of tropical field crops. Chichester, John Wiley \& Sons. p.231-248.

Fritsche-Neto R, Miranda GV, DeLima RO, Souza LV \& Silva J (2010) Herança de caracteres associados à eficiência de utilização do fósforo em milho. Pesquisa Agropecuária Brasileira, 45:465-471.

Hirel B, Le Gouis J, Ney B \& Gallais A (2007) The challenge of improving nitrogen use efficiency in crop plants: towards a more central role for genetic variability and quantitative genetics within integrated approaches. Journal of Experimental Botany, 58:2369-2387.

Hoagland DR \& Arnon DI (1938) The water culture method for growing plants without soil. Berkeley, California Agricultural Experiment Station. 347p.

Liu J, Chen F, Olokhnuud C, Glass ADM, Tong Y, Zhang F \& Mi G (2009) Root size and nitrogen-uptake activity in two maize (Zea mays) inbred lines differing in nitrogen-use efficiency. Journal of Plant Nutrition and Soil Science, 172:230-236.

Lopes AS, Daher E, Bastos ARR \& Guilherme LRG (2010) Suprimento e extensão das reservas de nutrientes no Brasil. In: Prochnow LI, Casarin V \& Stipp SR (Eds.) Boas práticas para uso eficiente de fertilizantes. Piracicaba, IPNI. p.283-307.

Machado CTT, Machado AT \& Furlani AMC (2004) Variação intrapopulacional em milho para características relacionadas com a eficiência de absorção e utilização de fósforo. Revista Brasileira de Milho e Sorgo, 3:77-91.

Malavolta E, Vitti GC \& Oliveira SA (1989) Avaliação do estado nutricional de plantas. Piracicaba, Potafos. 201p.

Moll RH, Kamprath EL \& Jackson A (1982) Analysis and interpretation of factors which contribute to efficiency of nitrogen utilization. Agronomy Journal, 74:562-564.

Montgomery DC \& Peck EA (1981) Introduction to linear regression analysis. New York, John Wiley \& Sons. 504p.

Murrel TS \& Fixen PE (2006) Improving fertilizer P effectiveness: challenges for the future. In: $3^{\text {rd }}$ International Symposium on Phosphorus Dynamics in the Soil-Plant Continuum, Uberlândia. Proceedings, Embrapa Milho e Sorgo. p.150-151.

Nielsen KL, Miller CR, Beck D \& Lynch JP (1999) Fractal geometry of root system: Fields observations of contrasting genotype of common bean (Phaseolus vulgaris L.). grown under different phosphorus regimes. Plant and soil, 206:181-190. 
Oliveira LR, Miranda GV, DeLima RO, Fritsche-Neto R \& Galvão JCC (2013) Eficiência na absorção e utilização de nitrogênio e atividade enzimática em genótipos de milho. Revista Ciência Agronômica, 44:614-621.

Parentoni SN, Mendes FF \& Guimarães LJM (2011) Melhoramento para eficiência no uso de fósforo. In: Fritsche-Neto R \& Borém A (Eds.) Melhoramento de plantas para condições de estresses abióticos. Visconde do Rio Branco, Editora Suprema. p.101-126.

SAS Institute Inc. (2005) Statistical Analysis user's guide. Version 9.1.3, Cary, Statistical Analysis System Institute. 220p

Taiz L \& Zeiger E (2009) Fisiologia vegetal. 4 ${ }^{\mathrm{a}}$ ed. Porto Alegre, Artmed. 848p.
Trachsel S, Messmer R, Stamp P \& Hund A (2009) Mapping of QTLs for lateral and axile root growth of tropical maize. Theoretical and Applied Genetics, 119:1413-1424.

Walk TC, Jaramillo R \& Lynch JP (2006) Architectural tradeoffs between adventitious and basal roots for phosphorus acquisition. Plant Soil, 279:347-366.

Wright S (1921) Correlation and causation. Journal of Agricultural Research, 20:557-585.

Zhu JM, Mickelson SM, Kaeppler SM \& Lynch JP (2006) Detection of quantitative trait loci for seminal root traits in maize (Zea mays L.) seedlings grown under differential phosphorus levels. Theoretical and Applied Genetics, 113:1-10. 